\title{
THE 2008 EXTREME OUTBURST OF THE YOUNG ERUPTIVE VARIABLE STAR EX LUPI
}

\author{
Colin Aspin $^{1}$, Bo Reipurth ${ }^{1}$, Gregory J. Herczeg ${ }^{2}$, and Peter Capak ${ }^{3}$ \\ ${ }^{1}$ Institute for Astronomy, University of Hawai'i at Manoa, 640 N. A'ohoku Place, Hilo, HI 96720, USA; caa@ifa.hawaii.edu, reipurth@ifa.hawaii.edu \\ ${ }^{2}$ Max-Planck-Institut für Extraterrestriche Physik, Giessenbachstrasse, 85748 Garching, Germany; gregoryh @ mpe.mpg.de \\ ${ }^{3}$ California Institute of Technology, MC 105-24, 1200 East California Boulevard, Pasadena, CA 91125, USA; capak@me.com \\ Received 2010 March 13; accepted 2010 June 28; published 2010 July 22
}

\begin{abstract}
In early 2008, the young low-mass star EX Lupi, the prototype of the EXor class of eruptive variables, optically brightened by over $5 \mathrm{mag}$ for a period of seven months. The previous time a change of such amplitude had been observed in EX Lup was over 50 years ago. In this Letter, we present new optical and near-IR highresolution spectroscopy of EX Lup during the 2008 outburst. We investigate the physical characteristics of the outburst both soon after it began and some four months later, and consider the observed energetics and kinematics. Emission line strengths, widths, and profiles significantly changed between the two observations. Also, modeling of the $2.2935 \mu \mathrm{m}$ CO overtone band head emission suggests that an inner gap in the circumstellar gas disk around the star may be present and that it is from the inner edge of the gas disk that the CO overtone emission probably arises. We derive a mass accretion luminosity and rate during the extreme outburst of $\sim 2 \pm 0.5 L_{\odot}$ and $\sim(2 \pm 0.5) \times 10^{-7} M_{\odot} \mathrm{yr}^{-1}$, respectively, which suggests that this outburst was indeed one of the strongest witnessed in EX Lup, yet not as strong as those observed in FU Orionis stars.
\end{abstract}

Key words: circumstellar matter - stars: formation - stars: individual (EX Lupi)

\section{INTRODUCTION}

Eruptive outbursts in young low-mass stars are scarce events that are thought to be the result of increased accretion through a circumstellar disk (Hartmann \& Kenyon 1996). EX Lupi is the prototype of a class of young stars (termed EXors) showing such outbursts (Herbig 1977, 1989, 2007). It has been observed to exhibit sporadic brightness increases of up to a few magnitudes, which typically last a few weeks before fading back to a quiescent state. Such a "characteristic outburst" occurred in 1993 and was studied spectroscopically by Lehmann et al. (1995) and Herbig et al. (2001). In contrast to these smaller characteristic outbursts, EX Lup was observed in 1955 to exhibit a larger outburst resulting in an optical brightness increase of $\sim 5$ mag. This event, which we herein term as an "extreme outburst," was until recently unique in the photometric annals of EX Lup. However, in early 2008 a second such event took place. Since it is unclear whether extreme outbursts are initiated by the same physical mechanism as their smaller more frequent counterparts, the advent of the 2008 eruption presented us with the opportunity to perform a detailed observational study of an extreme outburst. Below, we present the results of this investigation utilizing high spectral resolution optical (Keck I/ HIRES) and near-IR (Keck II/NIRSPEC) observations.

\section{OBSERVATIONS AND DATA REDUCTION}

Observations of EX Lup (16:03:05.5, -40:18:25, J2000) were made on the Keck I and II 10 m telescopes on UT 2008 January 23 (J23), 2008 February 23 (F23), and 2008 May 23 (M23). Two instruments were used: HIRES (Vogt et al. 1994) on Keck I and NIRSPEC on Keck II (McLean et al. 1995). Optical high-resolution spectra were obtained with HIRES on $\mathrm{J} 23$ and M23. On J23, the HIRESr configuration was used with the KV380 filter and a slit width of $0^{\prime \prime} 861$ resulting in a spectral resolution of $R \sim 48,000$ (i.e., a velocity resolution of $\sim 7 \mathrm{~km} \mathrm{~s}^{-1}$ ) and a wavelength coverage of $\sim 3850-8350 \AA$. On M23, the HIRESb configuration was used with the WG335 filter and the same slit resulting in a similar spectral resolution and a wavelength coverage of $3500-6300 \AA$. We are greatly indebted to G.H. Herbig for making the J23 HIRES spectra available. Near-IR (NIR) high-resolution spectra $(R \sim 18,000$ and velocity resolution $\sim 17 \mathrm{~km} \mathrm{~s}^{-1}$ ) were obtained with NIRSPEC on F23 and M23.

\section{RESULTS}

\subsection{Optical Light Curve}

Figure 1 shows the optical light curves of EX Lup over the 1955 (top), 1993 (middle), and 2008 (bottom) outbursts, taken from the AAVSO database. The 2008 outburst plot shows that the J23 HIRES observations were acquired soon after the extreme outburst occurred, some eight days after we had been notified of the event (A. Jones 2008, private communication to G.H. Herbig) and while the source was still in a rapid brightening phase with $\mathrm{m}_{V} \sim 10.5$ or $\sim 3.5$ mag brighter than in quiescence $\left(m_{V} \sim 14\right)$. Our first NIRSPEC observations were taken one month later when EX Lup had passed its peak brightness of $m_{V} \sim 8$ and had $m_{V} \sim 9.5$. Our second set of HIRES and NIRSPEC observations were taken simultaneously three months later (M23), during the relatively constant "plateau phase" of the outburst which lasted $\sim 170$ days. At this time, EX Lup had $m_{V} \sim 10.2$ and was close to the start of a period of instability, about two months prior to the sharp decline back to quiescence. We note that both the 1955 and 2008 extreme outbursts lasted approximately the same length of time although they are somewhat different in behavior; the 1955 eruption started slowly and built up to a maximum (after $\sim 160$ days) while the 2008 event appeared to have a more rapid rise to maximum. The light curve suggests that the 1993 event was a smaller characteristic outburst.

\subsection{HIRES Spectra}

In Figure 2, we show three wavelength regions from the $\mathbf{J} 23$ HIRES spectrum, selected to cover important atomic features, 

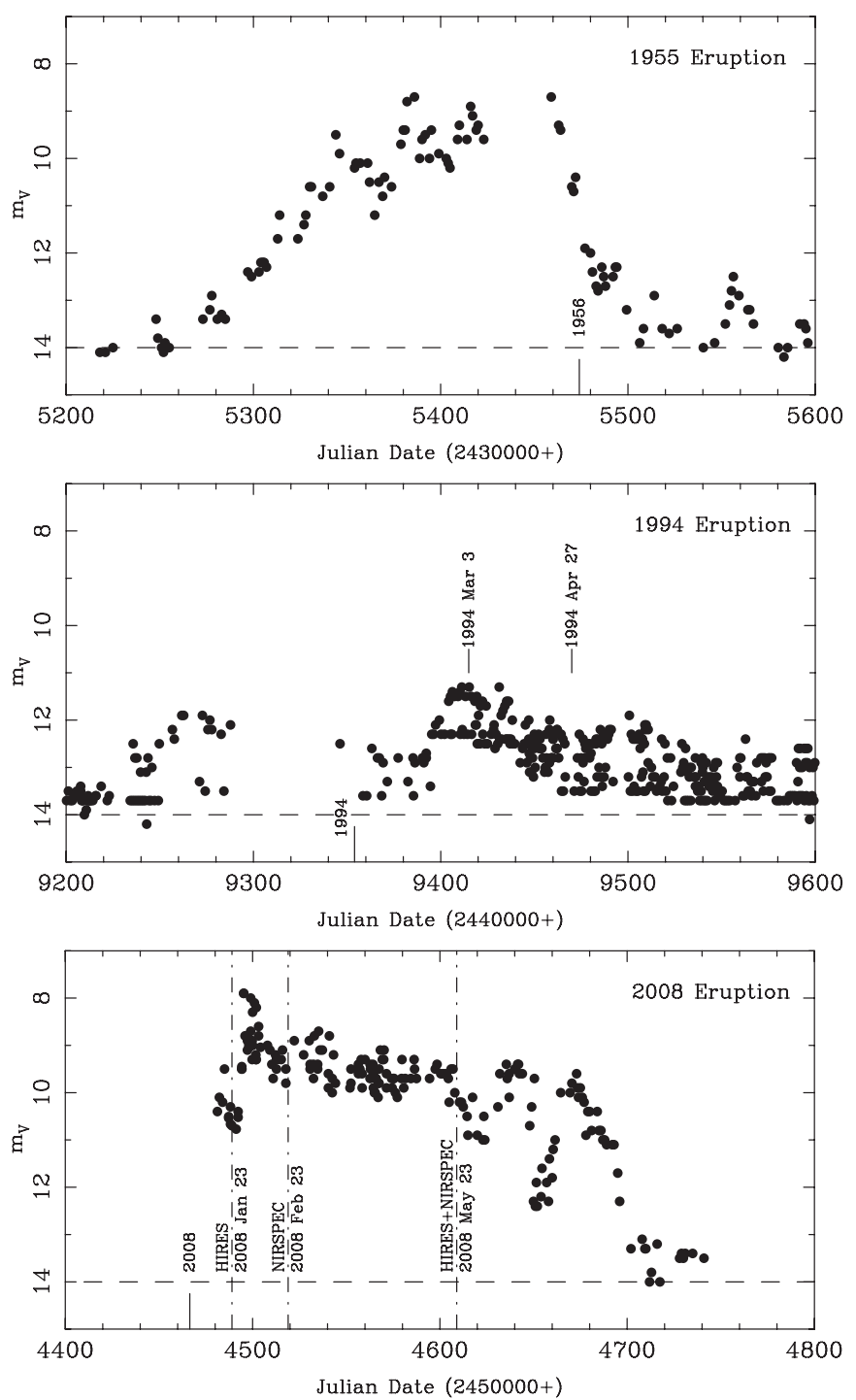

Figure 1. Optical light curves during the 1955 (top), 1993 (middle), and 2008 (bottom) outbursts. All plots cover 400 days starting from shortly before the outbursts began. The mean "quiescent" optical brightness of EX Lup is indicated by the horizontal dashed lines. All data points are taken from the AAVSO database. The vertical dot-dashed lines in the bottom plot indicate the times when the HIRES and NIRSPEC spectroscopic observations were acquired. The dates shown in the middle plot are those when the Lehmann et al. (1995) optical spectra were taken.

specifically, $\mathrm{H} \beta$ (4861.3 $\AA$, top), $\mathrm{He}_{\mathrm{I}}(5875.7 \AA$ ) $)$ and $\mathrm{Na}$ I (5890.0 and 5895.9 $\AA$, middle), and He I (6678.2 $\AA$ ) and Li I (6707.8 ̊, bottom). All but Li I are seen in emission (which is seen in absorption). Na I and $\mathrm{H} \beta$ both possess blueshifted absorption, with the emission lines being broad (full width $10 \%$ intensity $\sim 750 \mathrm{~km} \mathrm{~s}^{-1}$ ) and the blueshifted absorption removing flux well below the continuum with a minimum at $\sim-120 \mathrm{~km} \mathrm{~s}^{-1}{ }^{4}$ The blueshifted absorption on $\mathrm{H} \beta$ appears smooth with one broad minimum at $-115 \mathrm{~km} \mathrm{~s}^{-1}$. Similarly, the blueshifted absorption on the Na I D lines have minima at -129 and $-122 \mathrm{~km} \mathrm{~s}^{-1}$, respectively. Fe I, Fe II, and He I all exhibit composite profiles made up of both broad and narrow emission components (BC and NC, respectively; Hamann \& Persson 1992). The NC and BC peaks lie within $\sim 10 \mathrm{~km} \mathrm{~s}^{-1}$ of their rest wavelength.

\footnotetext{
4 All radial velocities are relative to the EX Lup rest velocity. EX Lup has $v_{\text {helio }}=+23,+28$, and $+2 \mathrm{~km} \mathrm{~s}^{-1}$, on J23, F23, and M23, respectively.
}
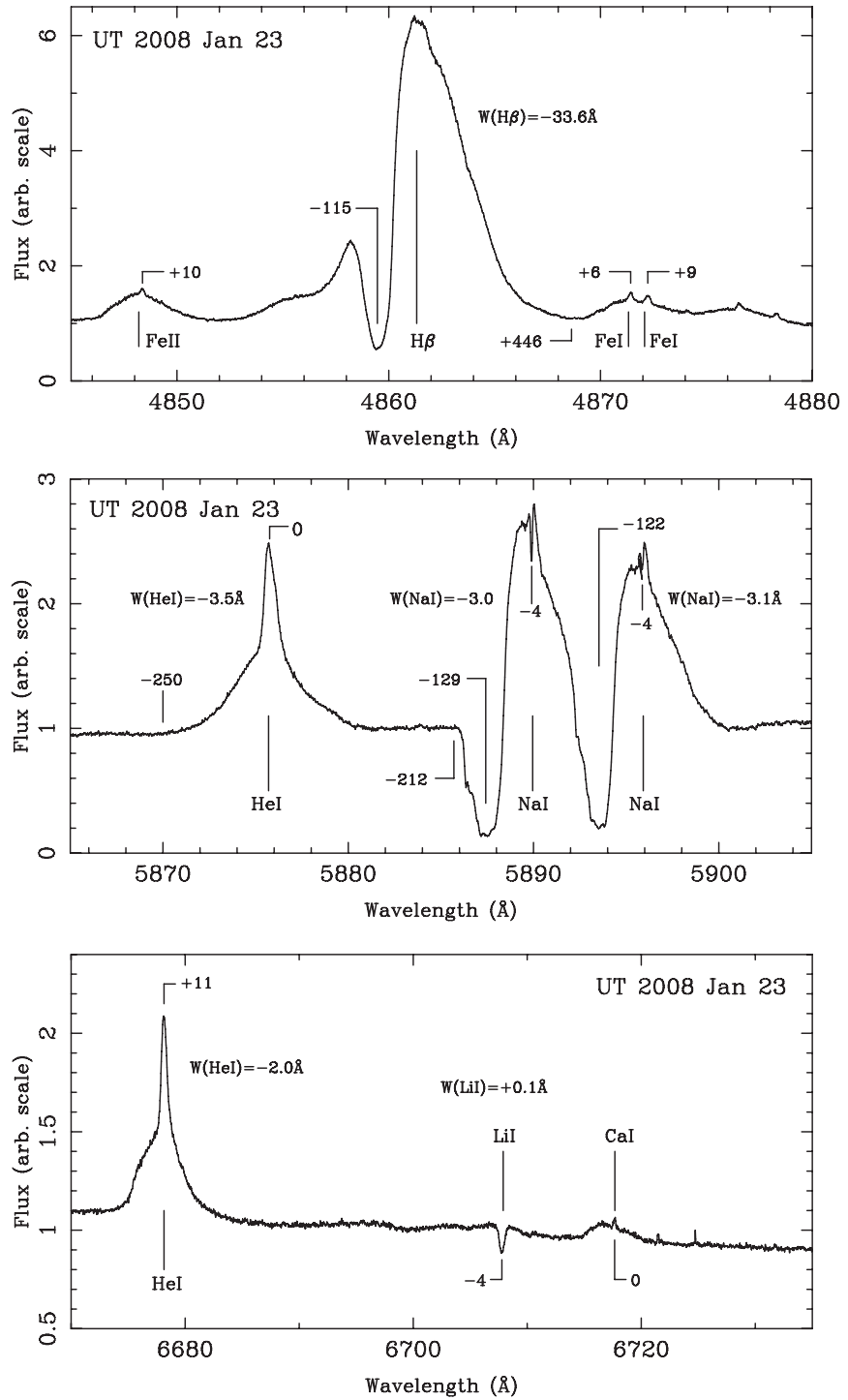

Figure 2. Optical HIRES spectra taken on UT 2008 January 23 close to the start of the outburst. Three wavelength regions are shown containing $\mathrm{H} \beta$ (top), $\mathrm{He} \mathrm{I}$ and $\mathrm{Na}$ I (middle), and He I and Li I (bottom). Heliocentric radial velocities are given for the major features of the atomic lines. Equivalent widths $(W)$ of the important features are shown.

Four months after the first HIRES observation, on M23 (see Figure 3), much of the same spectral structure was still visible although some changes appeared to have occurred. ${ }^{5}$ The He I line still possessed $\mathrm{NC}$ and $\mathrm{BC}$ features although the radial velocity offset of the NC from the line rest wavelength had shifted to the blue. Also, the $\mathrm{H} \beta$ profile had changed and exhibited a double peak at -77 and $+100 \mathrm{~km} \mathrm{~s}^{-1}$ with an absorption minimum at $+42 \mathrm{~km} \mathrm{~s}^{-1}$. However, the width of the $\mathrm{H} \beta$ emission was similar to that found four months previously. The profiles of the Na I D lines were also quite different and became somewhat more complex. Both lines remained in emission and possessed blueshifted absorption components although the latter were much weaker than in the J23 spectra. Additionally, the blueshifted absorptions showed radial velocity structure (see Figure 3) extending to $-277 \mathrm{~km} \mathrm{~s}^{-1}$ on M23 and $-212 \mathrm{~km} \mathrm{~s}^{-1}$ on J23. Finally, superimposed on both of the broad

\footnotetext{
5 In the M23 observations, Li I (6707.8 $)$ and He I (6678.2 $)$ did not fall on
} the detector. 

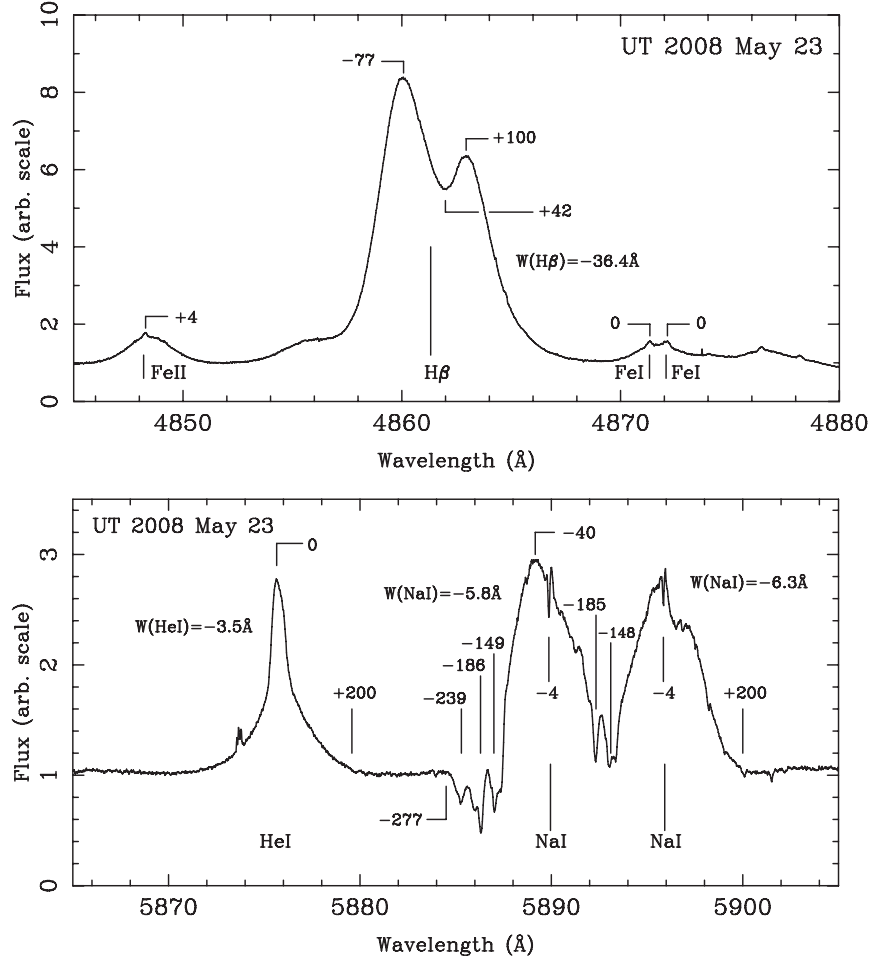

Figure 3. Optical HIRES spectra taken on UT 2008 May 23 some four months after the outburst began. Two of the three wavelength regions presented in Figure 2 are shown here. The HIRES echelle order containing the $6707 \AA$ Li I line was not observed on this date due to the different echelle setting used.

$\mathrm{Na}$ I emission lines were narrow absorption features with a radial velocity offset of $-4 \mathrm{~km} \mathrm{~s}^{-1}$.

We have performed a best fit to the NC and BC structures present in He I and Fe II using a two-component Gaussian emission profile model, iterating over three parameters for both Gaussians, specifically, the wavelength of peak emission $\left(\lambda_{\text {peak }}\right)$, the peak flux $\left(F_{\text {peak }}\right)$, and the Gaussian FWHM. The results of this procedure are shown in Figure 4. Between the two dates we find that (1) for both lines $\lambda_{\text {peak }}$ of the NC and BC Gaussians remained within $\sim 10 \mathrm{~km} \mathrm{~s}^{-1}$ of the line rest wavelength, (2) the He I NC FWHM did not change significantly, remaining at $\sim 36 \mathrm{~km} \mathrm{~s}^{-1}$, while the Fe II NC FWHM became smaller by a factor of 2 (14 and $7 \mathrm{~km} \mathrm{~s}^{-1}$ ) and at both times was significantly smaller than the He I NC FWHM, (3) the He I BC FWHM declined (from 220 to $180 \mathrm{~km} \mathrm{~s}^{-1}$ ), while the Fe II BC FWHM remained around the same (at $\sim 170 \mathrm{~km} \mathrm{~s}^{-1}$ ), and (4) the He I $F_{\text {peak }}$ of both the NC and BC increased slightly (by $\sim 20 \%$ ). The He $\mathrm{I}$ equivalent width $(\mathrm{EW})$ on the two dates was $\mathrm{EW}\left(\mathrm{He}_{\mathrm{I}}\right)=$ $-3.5 \AA\left(\mathrm{J} 23: \mathrm{EW}_{\mathrm{NC}}=-0.69 \AA, \mathrm{EW}_{\mathrm{BC}}=-2.80 \AA ; \mathrm{M} 23: \mathrm{EW}_{\mathrm{NC}}\right.$ $\left.=-0.82 \AA, \mathrm{EW}_{\mathrm{BC}}=-2.70 \AA\right)$. Similarly, $\mathrm{EW}(\mathrm{Fe}$ II $)$ on J23 was $-5.8 \AA\left(\mathrm{EW}_{\mathrm{NC}}=-0.21 \AA, \mathrm{EW}_{\mathrm{BC}}=-5.60 \AA\right)$ and on $\mathrm{M} 23$ was $-7.9 \AA\left(\mathrm{EW}_{\mathrm{NC}}=-0.10 \AA, \mathrm{EW}_{\mathrm{BC}}=-7.80 \AA\right)$.

\subsection{NIRSPEC Spectra}

Data for three spectral regions taken from the NIRSPEC observations are shown in Figure 5. Here, results from both observing dates (F23 and M23) are plotted for the regions covering $\mathrm{Br} \gamma$ at $2.166 \mu \mathrm{m}$ (top left) and the $v=2-0 \mathrm{CO}$ overtone band head at $2.2935 \mu \mathrm{m}$ (top right). The bottom left plot covers the Na I doublet lines at 2.206 and $2.208 \mu \mathrm{m}$ from F23 only. ${ }^{6}$ The Bry line appears very similar in J23 and M23

\footnotetext{
6 The Na I spectral region was not included in the M23 data set.
}

with an $\mathrm{EW}(\mathrm{Br} \gamma)$ of $-17 \AA$. It is broad $\left(\mathrm{FWHM} \sim 220 \mathrm{~km} \mathrm{~s}^{-1}\right.$ on $\mathrm{F} 23,190 \mathrm{~km} \mathrm{~s}^{-1}$ on M23) and is slightly redshifted with the $\lambda_{\text {peak }}$ emission offset by +5 and $+32 \mathrm{~km} \mathrm{~s}^{-1}$ on F23 and M23, respectively. The Na I doublet lines are also broad with FWHM of 177 and $155 \mathrm{~km} \mathrm{~s}^{-1}$ on the one date that they were observed. The most striking change occurs in the spectral region containing the $2.2935 \mu \mathrm{m} v=2-0 \mathrm{CO}$ overtone band head. In the data from F23, the band head is strongly in emission with an $\mathrm{EW}(\mathrm{CO})=-21 \AA^{7}$ and exhibits the characteristic signature of being significantly velocity broadened (i.e., a "blue hump" is present; see, for example, WL 16 in Carr et al. 1993). In the data from M23, however, the emission is only barely seen $(\mathrm{EW}(\mathrm{CO})=-4 \AA$ ) although velocity broadening of the band head still seems to be present. In the bottom right plot of Figure 5, we have modeled the $\mathrm{CO}$ band head emission profile using the analysis from Dent \& Geballe (1991) and Carr \& Tokunaga (1992) previously employed in Aspin et al. (1994) and Aspin $\&$ Greene (2007). The details of the model are presented in the aforementioned papers and, for brevity, here we merely present our findings. The $\mathrm{CO}$ band head emission is best fitted using a velocity profile originating in a circumstellar disk. This produces a good fit to the blue hump and the overall shape of the band head. We investigated a wide range of the model free parameters, specifically stellar mass $\left(M_{*}\right)$ over the range $0.1-2.0 M_{\odot}$, the emission region temperature $\left(T_{\mathrm{CO}}\right)$ from 1500 to $6000 \mathrm{~K}$, the optical depth of $\mathrm{CO}\left(\tau_{\mathrm{CO}}\right)$ from 0.01 to 5.0 , and the inner $\left(r_{\text {in }}\right)$ and outer $\left(r_{\text {out }}\right)$ gas disk radii between $1.0 \times 10^{9}$ and $1.0 \times$ $10^{11} \mathrm{~m}$ (i.e., $\sim 1.4-140 R_{*}$ or $\left.0.007-0.7 \mathrm{AU}\right)$. The best fit to the spectrum gave values of $M_{*}=0.6 M_{\odot}, T_{\mathrm{CO}}=2500 \mathrm{~K}, \tau_{\mathrm{CO}}<$ $0.1, r_{\text {in }}=1.2 \times 10^{10} \mathrm{~m}\left(\sim 17 R_{*}\right.$ or $\left.0.08 \mathrm{AU}\right)$, and $r_{\text {out }}=2.0 \times$ $10^{10} \mathrm{~m}\left(\sim 29 R_{*}\right.$ or $\left.0.13 \mathrm{AU}\right)$. Typical associated uncertainties on these values should be considered to be $\sim 20 \%$. For this value of $r_{\text {in }}$ and with $R_{*}=1.6 R_{\odot}$ (as estimated by Gras-Velázquez \& Ray 2005 and Sipos et al. 2009), gas at $2500 \mathrm{~K}$ would require a heating source on the stellar surface of $\sim 8000 \mathrm{~K}$ similar to what was found for surface accretion shocks in, for example, V1647 Ori (Briceño et al. 2004). The Keplerian velocity at 0.08 AU for $M_{*}=0.6 M_{\odot}$ is $\sim 80 \mathrm{~km} \mathrm{~s}^{-1}$. Due to the complex relationship between the model free parameters, the uniqueness of the above best-fit model is unclear. However, we believe that our investigation of the aforementioned free-parameter space results in values for the above physical properties that are reasonable for this star.

\section{DISCUSSION}

\subsection{Photometric Variability}

The 2008 extreme outburst of EX Lup appears to be unlike any other observed in this source since 1955 . Both extreme outbursts exhibited very large optical brightness increases (5-6 mag) and, although information about the earlier event is scarce, they appear, at least superficially, to be quite similar. Between 1955 and 2008, EX Lup has undergone numerous smaller characteristic outbursts with $\Delta m_{V}$ typically 1-3 mag. The durations of the extreme and characteristic events appear very different; the former last 200+ days whereas the latter last perhaps only a few weeks (see the AAVSO archive for more details). Photometrically, therefore, there seems to be an inherent difference between extreme and characteristic outbursts.

\footnotetext{
Calculated from 2.2925 to $2.2975 \mu \mathrm{m}$.
} 

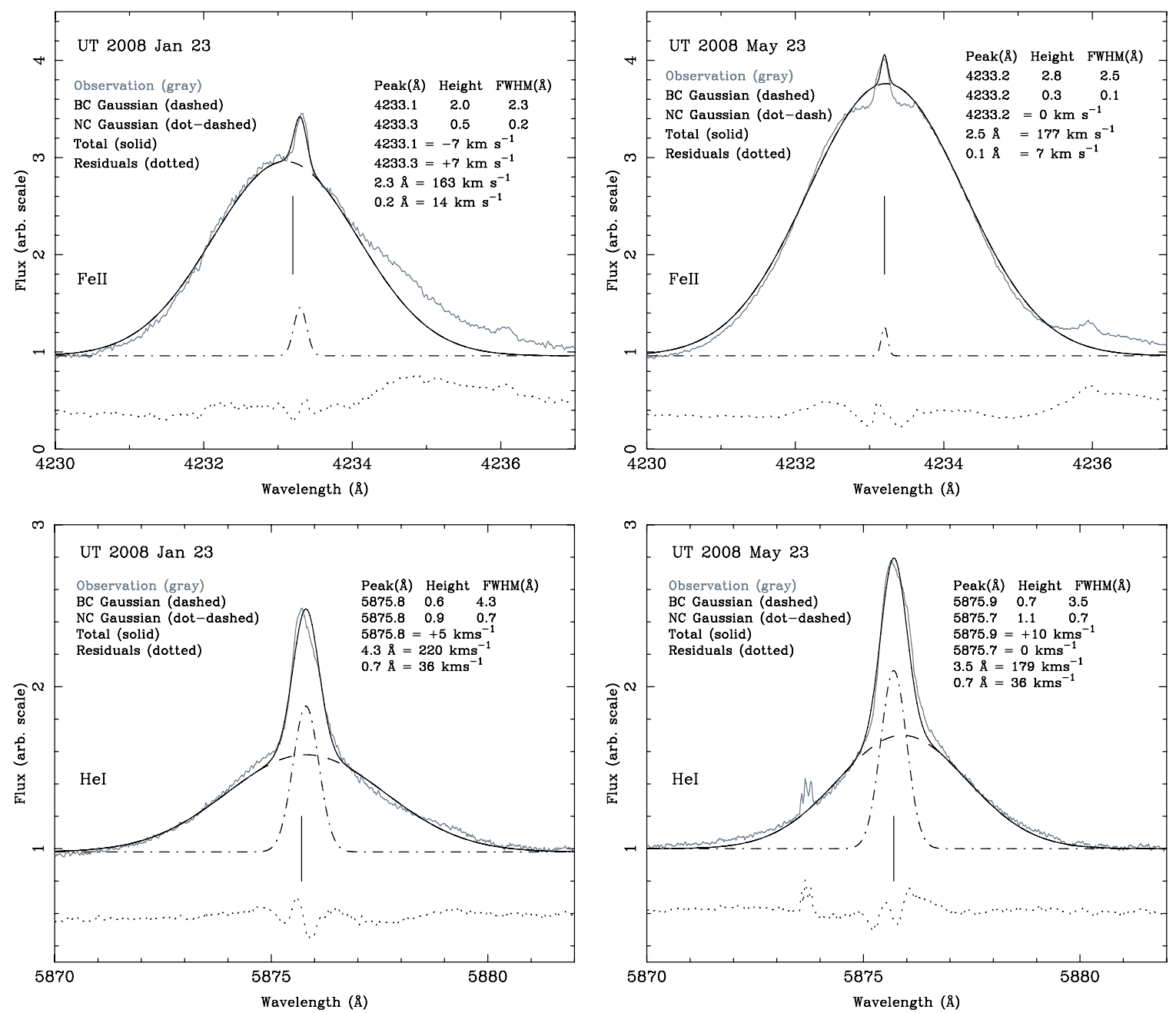

Figure 4. HIRES spectra of the Fe II line at $4233.2 \AA$ (top, gray lines) and the He I line at $5876.1 \AA$ (bottom, gray lines) overlaid with model Gaussian profiles. The best-fit parameters are given in the legends. The NC Gaussian is shown as dot-dashed lines while the BC Gaussian is shown as dashed lines. The total model profile is the solid (black) lines and the residuals between the observed and model profiles are shown by the dotted lines. The heliocentric rest wavelength of the line is shown as a vertical solid line.

\subsection{Spectroscopic Variability}

The HIRES spectra of EX Lup taken between 1998 and 2004 (presented in Herbig 2007) and covering both the quiescent state and the characteristic outburst phases, showed narrow emission features together with an underlying atomic and molecular absorption spectrum typical of an $\sim \mathrm{M} 0$ dwarf star. During the 2008 extreme outburst, however, the HIRES spectra appeared markedly different with no obvious sign of an underlying photosphere. Comparing our data taken during the 2008 extreme outburst (shown in Figures 2 and 3) with previous observations, we find that whereas Herbig et al. (2001) found strong emission lines of $\mathrm{HI}, \mathrm{He}$ I, He II, and Ca II with no evidence for blueshifted absorption nor Fe I and Fe II lines, we observe both. Also, the characteristic outburst phase spectrum from Lehmann et al. (1995) possessed H I, He I, He II, and Ca II lines together with weak Fe II lines, exhibiting inverse P Cygni (IPC) profiles (redshifted absorption) on $\mathrm{H}$ I, Ca II, and perhaps even on Fe II which disappeared around seven weeks later. Herbig (1950) noted that IPC profiles were also present in his 1949/1950 spectrograms taken several years before the earlier extreme outburst. Lehmann et al. (1995) interpreted these IPC profiles as originating in cool infalling gas. Our HIRES spectra, which show blueshifted absorption, suggest that we are observing the opposite, i.e., cool outflowing gas. We speculate that perhaps it is only during extreme outbursts that strong and dense enough stellar/disk winds are generated to create the blueshifted absorption component.

Two-component (BC and NC) emission line profiles, similar to the ones seen in our 2008 EX Lup spectra, were previously reported on the stronger Fe II and Ti II lines in EX Lup in data taken in 1998 June (Herbig 2007). Another young T Tauri star to show such structure is DR Tau (Beristain et al. 1998). The interpretation suggested for this source was that the NC of the lines arose in a compact region close to the stellar surface (and hence not significantly rotationally broadened) as in a postshock region at the base of an accretion flow. The $\mathrm{BC}$ was considered to originate in a region with considerable radial velocity structure such as in a magnetospheric accretion funnel or in the hot inner region of the circumstellar disk. The different excitation requirements for $\mathrm{He}$ I $\left(E_{\text {upper }}=23 \mathrm{eV}\right)$ and $\mathrm{Fe}$ II $\left(E_{\text {upper }}=5-6 \mathrm{eV}\right)$ suggest that these lines are created in different physical locations, making the similarity of structure somewhat peculiar. Any model proposed to explain the formation of these lines and the creation of the $\mathrm{NC}+\mathrm{BC}$ structure would need to explain this energy discrepancy together with the temporal 

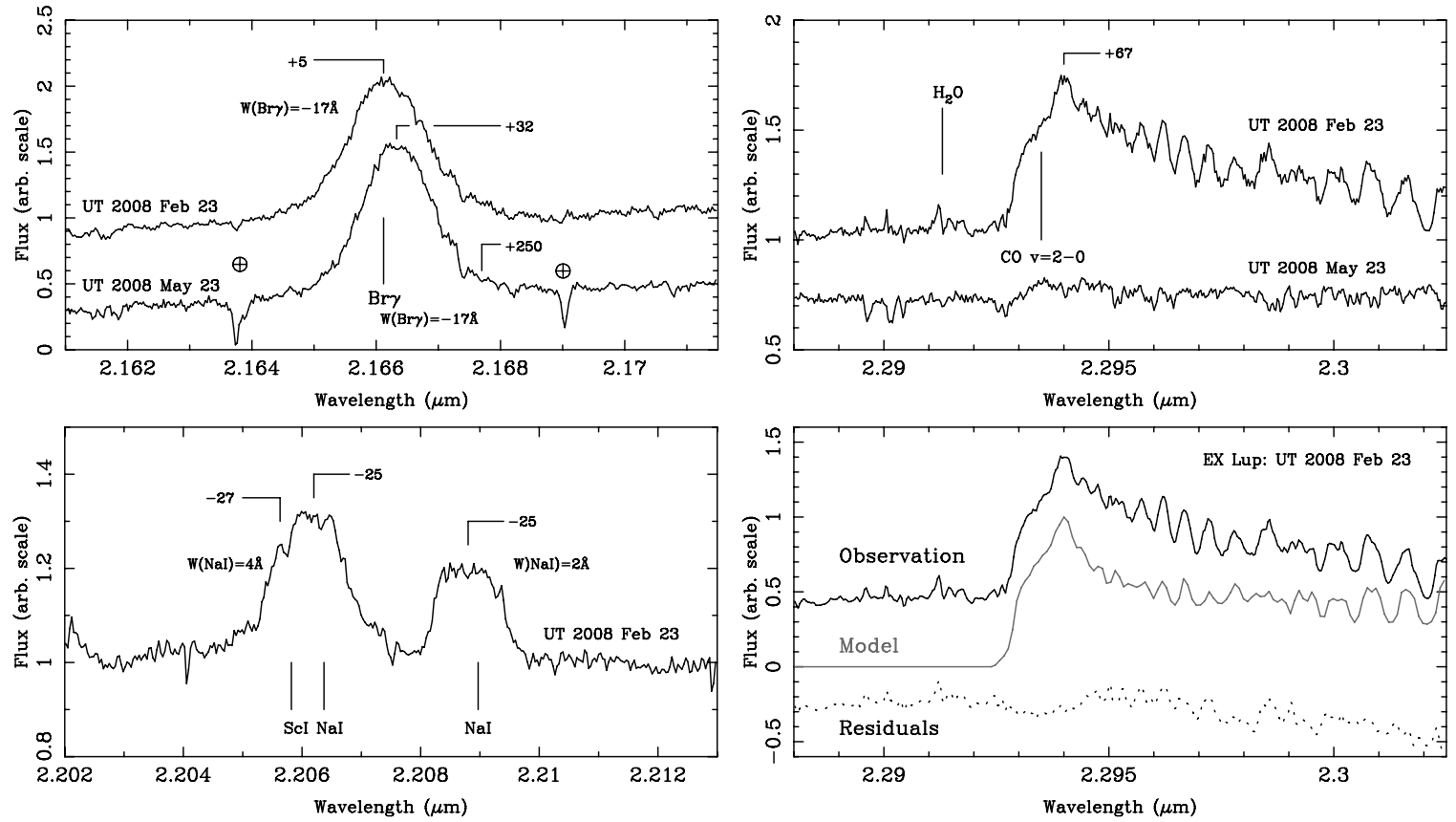

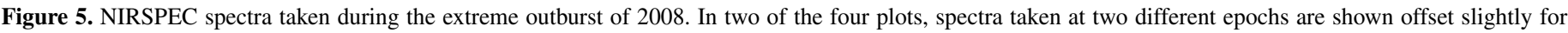

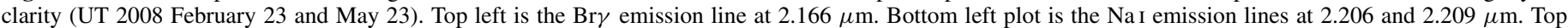

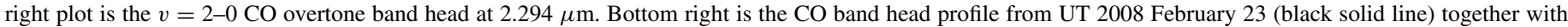

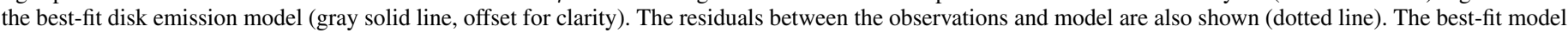
parameters are described in the text.

changes observed between $\mathrm{J} 23$ and M23, i.e., (1) a decline in the strength (i.e., EW) of the Fe II NC by a factor of 2 coupled with an $\sim 40 \%$ increase in the BC strength, (2) an $\sim 20 \%$ increase in the strength of the He I NC coupled with an unchanged BC strength, and (3) the changes in kinematic properties (e.g., FWHM, radial velocity offset) shown in Figure 4.

The changes in the blueshifted absorption profiles between J23 and M23 suggest that the wind from EX Lup evolved between the two observations. The blueshifted absorption features present on $\mathrm{J} 23$ and seen in the $\mathrm{H}, \mathrm{Na}$, and $\mathrm{Ca}$ lines, varied somewhat differently for each element. Between J23 and M23, (1) the blueshifted $\mathrm{H} \beta$ absorption moved redward and, in fact, became somewhat redshifted, (2) the blueshifted Ca I absorption disappeared, and (3) the deep and broad single component blueshifted absorption on the Na I lines became shallower and more complex in structure and extended to much higher radial velocities. A sophisticated radiative transfer code is required to model such variations.

The changes in the $\mathrm{CO}$ overtone band head observed between $\mathrm{J} 23$ and M23, together with the modeling presented above, suggest that the hot gas in the inner regions of the accretion disk either cooled significantly between the two dates, or was mostly accreted onto the star, or the UV photoexcitation conditions changed (see Brittain et al. 2009). The unchanged Br $\gamma$ flux over this period implies strong on-going accretion.

\subsection{Accretion Luminosity and Rate}

On J23, F23, and M23, EX Lup had $\mathrm{m}_{V}$ values of 10.5, 9.5, and 10.2, respectively. A. Juhasz et al. (2010, in preparation) measured the $K$-band magnitude of EX Lup to be $\mathrm{m}_{K}=7.5$ on UT 2008 April 20. On that date EX Lup had a $V$ magnitude of $\sim 9.5$ and hence a $V-K=2.0$. If we assume this $V-K$ value for F23 and M23 then on those dates $m_{K}$ was 7.5 and
8.2, respectively (a mean of 7.85). The $\mathrm{Br} \gamma$ emission line fluxes therefore decreased by two times between J23 and M23 since on both dates the $\mathrm{EW}$ of $\mathrm{Br} \gamma$ was $-17 \AA$. If the optical continuum emission and the hydrogen line fluxes are indicative of the mass accretion rate (e.g., Gullbring et al. 1998; Muzerolle et al. 1998a, 1998 b), then the accretion rate changed by only a factor of 2 over this four month period.

We can convert line and continuum luminosities to accretion luminosity $\left(L_{\text {acc }}\right)$ to estimate the mass accretion rate $(\dot{M})$. Following Aspin et al. (2008) and adopting $m_{K}=7.85$, a flux corresponding to zeroth magnitude at $2.2 \mu \mathrm{m}$ of $4.35 \times$ $10^{-10} \mathrm{~W} \mathrm{~m}^{-2} \mu \mathrm{m}^{-1}$ (Tokunaga \& Vacca 2005), a visual extinction $A_{V} \sim 0, r_{\text {in }}=17 R_{*}$ (see above), and $L_{*}=0.5 L_{\odot}$ (derived from $T_{*}=3800 \mathrm{~K}$ and $R_{*}=1.6 R_{\odot}$, again see above), and a distance of $140 \pm 20 \mathrm{pc}$ (Hughes et al. 1993), we obtain values of $L_{\text {acc }} 1.6 \pm 0.6 L_{\odot}$ and $\dot{M}=(1.5 \pm 0.5) \times 10^{-7} M_{\odot} \mathrm{yr}^{-1}$. Following Valenti et al. (1993) and Gullbring et al. (1998), the change in optical continuum emission in extreme outburst $(\Delta V=4.5 \mathrm{mag})$ is converted into $L_{\text {acc }} \sim 2.3 L_{\odot}$ and $\dot{M} \sim 2.1 \times 10^{-7} M_{\odot} \mathrm{yr}^{-1}$. We therefore adopt extreme outburst values of $L_{\text {acc }}=$ $2 \pm 0.5 L_{\odot}$ and $\dot{M}=(2 \pm 0.5) \times 10^{-7} M_{\odot} \mathrm{yr}^{-1}$. Sipos et al. (2009) estimated the quiescent accretion luminosity and rate of EX Lup to be $L_{\text {acc }} \sim 0.0037 L_{\odot}$ and $\dot{M} \sim 4.0 \times 10^{-10} M_{\odot} \mathrm{yr}^{-1}$ using $\mathrm{Pa} \beta$ line fluxes. Since EX Lup is veiled even in quiescence, ${ }^{8}$ we adopt veiling-corrected values for quiescent $L_{\text {acc }}$ and $\dot{M}$ of $0.05 \pm 0.02$ and $(6 \pm 3) \times 10^{-9}$, respectively.

The accretion luminosity and rate during the 2008 extreme outburst of EX Lup are therefore $\sim 40 \times$ larger than their quiescent values. However, these increases are still $\sim 50 \times$ smaller than those found in FU Orionis eruptions (Hartmann \& Kenyon 1996).

\footnotetext{
8 Herbig (2007) estimated an optical veiling of $\sim 1$ when EX Lup was faint.
} 
In conclusion, the 2008 extreme outburst of EX Lup possessed a number of features that distinguished it from the more common characteristic outbursts and appears to have had more in common with the previous extreme outburst that occurred in 1955.

We warmly thank G.H. Herbig for providing access to the J23 HIRES data on EX Lupi. We are indebted to A. Jones for alerting us (via G.H. Herbig) to this new outburst of EX Lup. We also thank A. Juhasz for providing the $K$-band magnitude used above. Data were obtained at the W.M. Keck Observatory, operated as a scientific partnership by the California Institute of Technology, the University of California, and the National Aeronautics and Space Administration. B.R. acknowledges partial support from the NASA Astrobiology Institute (Cooperative Agreement No. NNA04CC08A).

\section{REFERENCES}

Aspin, C., \& Greene, T. P. 2007, AJ, 133, 568

Aspin, C., Beck, T. L., \& Reipurth, B. 2008, AJ, 135, 423

Aspin, C., Reipurth, B., \& Lehmann, T. 1994, A\&A, 288, 165

Beristain, G., Edwards, S., \& Kwan, J. 1998, ApJ, 499, 828
Briceño, C., et al. 2004, ApJ, 606, L123

Brittain, S. D., Najita, J. R., \& Carr, J. S. 2009, ApJ, 702, 85

Carr, J. S., \& Tokunaga, A. T. 1992, ApJ, 393, L67

Carr, J. S., Tokunaga, A. T., Najita, J., Shu, F. H., \& Glassgold, A. E. 1993, ApJ, 411, L37

Dent, W. R. F., \& Geballe, T. R. 1991, A\&A, 252, 775

Gras-Velázquez, Á., \& Ray, T. P. 2005, A\&A, 443, 541

Gullbring, E., Hartmann, L., Briceno, C., \& Calvet, N. 1998, ApJ, 492, 323

Hamann, F., \& Persson, S. E. 1992, ApJS, 82, 247

Hartmann, L., \& Kenyon, S. J. 1996, ARA\&A, 34, 207

Herbig, G. H. 1950, PASP, 62, 211

Herbig, G. H. 1977, ApJ, 217, 693

Herbig, G. H. 1989, in Workshop on Low Mass Star Formation and Pre-main Sequence Objects, ed. B. Reipurth (ESO: Garching), 233

Herbig, G. H. 2007, AJ, 133, 2679

Herbig, G. H., Aspin, C., Gilmore, A. C., Imhoff, C. L., \& Jones, A. F. 2001, PASP, 113, 1547

Hughes, J., Hartigan, P., \& Clampitt, L. 1993, AJ, 105, 571

Lehmann, T., Reipurth, B., \& Brandner, W. 1995, A\&A, 300, L9 (LRB95)

McLean, I. S., Becklin, E. E., Figer, D. F., Larson, S., Liu, T., \& Graham, J. 1995, Proc. SPIE, 2475, 350

Muzerolle, J., Calvet, N., \& Hartmann, L. 1998a, ApJ, 492, 743

Muzerolle, J., Hartmann, L., \& Calvet, N. 1998b, AJ, 116, 2965

Sipos, N., Ábrahám, P., Acosta-Pulido, J., Juhász, A., Kóspál, Á., Kun, M., Moór, A., \& Setiawan, J. 2009, A\&A, 507, 881

Tokunaga, A., \& Vacca, W. D. 2005, PASP, 117, 1459

Valenti, J. A., Basri, G., \& Johns, C. M. 1993, AJ, 106, 2024

Vogt, S. S., et al. 1994, Proc. SPIE, 2198, 362 